\title{
Comparative Study of Different Technique for Medical Image Segmentation: A Survey
}

\author{
AbhishekChandale,Divakarsingh \\ B-UIT Bhopal India \\ abhi.uitrgpv@gmail.com \\ B-UIT Bhopal IndiaHead, \\ divakar_singh@rediffmail.com
}

\begin{abstract}
Image analysis and processing can be done using image segmentation as one of the most critical aspect. Varioussubsequent processes of image analysis such as object representation object description, feature measurement get affected as a consequence of segmentation. Other higher level tasks such as object classification also get affected $b$ y segmentation. Due to all above reasons, imagesegmentation is considered as one of the most vital and essential process for providing features like delineration, characterization and visualization of important regions in any medical image. Its is necessary to provide accuracy with less time consumption in medical image segmentation Since manual segmentation is very tedious and time consuming to achieve and medical images are mostly unmanageable to be examined .Hence it is necessary to analysis \& review current methods and algorithms accruing and need minimal user interaction in case of medical images. In medical images segmentation, the anatomical structure or region to be examined has to be extracted and delineated so as to improve its visibility. This paper projects importance of decision making process in segmentation of images to extract relevant information.
\end{abstract}

Keywords Terms: - Medical image segmentation, image analysis.

\section{Council for Innovative Research}

Peer Review Research Publishing System

Journal: INTERNATIONAL JOURNAL OF COMPUTERS \& TECHNOLOGY

Vol 11, No. 1

editor@cirworld.com

www.cirworld.com, member.cirworld.com 


\section{Introduction}

For medical diagnosis and treatment of any patient , medical images play and extremely important role in assisting and guiding health care providers .Interpretation of medical images solely depends on radiologists, however this consumes reasonable time and the accuracy depends on experience of the radiologist. Various A I methodologies such as digital image processing when combined with others like machine learning, fuzzy logic and pattern recognition are so valuable in Image techniques can be grouped under a general framework; Image Engineering (IE). This is comprised of three layers: image processing (lower layer), image analysis (middle layer), and image understanding (high layer), as shown in Fig 1. Image segmentation is shown to be the first step and also one of the most critical tasks of image analysis. Its objective is that of extracting information (represented by data) from an image via image segmentation, object representation, and feature measurement, as shown in Fig 1. Result of segmentation; obviously have considerable influence over the accuracy of feature measurement [1]. The computerization of medical image segmentation plays an important role in medical imaging applications. It has found wide application in different areas such as diagnosis, localization of pathology, study of anatomical structure, treatment planning, and computer-integrated surgery. However, the variability and the complexity of the anatomical structures in the human body have resulted in medical image segmentation remaining a hard problem [2].

\section{Methods}

In this section, we briefly describe several common approaches that have appeared in the recent literature on medical image segmentation. We define each method, provide an overview of how the method is implemented, and discuss its limitations.

We divide segmentation methods into SOME categories: approaches

Regions based approaches region growing approaches classifiers clustering approaches, Markov random field models artificial neural networks Edge detection Technique

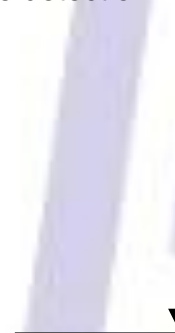

Voxel based Thresolding linear filters Histogram based Mothod

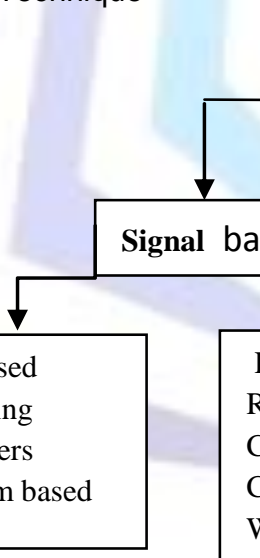

Signal based
Region based Region Growing Connected Components Watershed Splits\& merge Non-Linear filters Image Segmentation

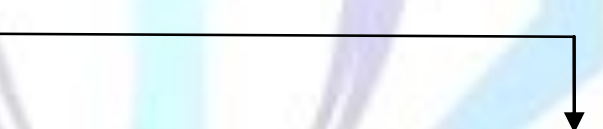

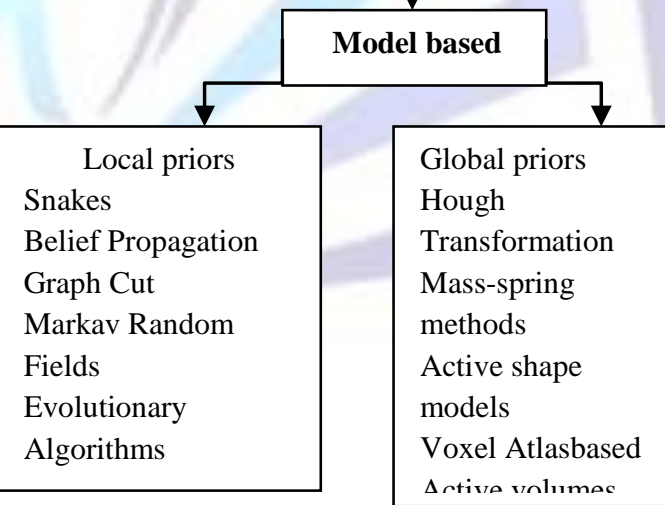

Figure 1:Different Image segmentation technique

\subsection{Thresholding}

approaches segment scalar images by creating a binary partitioning of the image intensities. A procedure attempts to determine an intensity value, called the threshold, which separates the desired classes. The segmentation is then achieved by grouping all pixels with intensity greater than the threshold into one class, and all other pixels into another class. Two potential thresholds are shown in Figure $2 \mathrm{a}$ at the valleys of the histogram. Determination of more than one threshold value is a process called multi[3] 


\subsection{Segmentation Based On Edge Detection}

This method attempts to resolve image segmentation by detecting the edges or pixels between different regions that have rapid transition in intensity are extracted [6,5] and linked to form closed object boundaries. The result is a binary image. Based on theory there are two main edge based segmentation methods- gray histogram and gradient based method [4].

Edge detection is a well-developed field on its own within image processing. Region boundaries and edges are closely related, since there is often a sharp adjustment in intensity at the region boundaries. Edge detection techniques have therefore been used as the base of another segmentation technique. The edges identified by edge detection are often disconnected. To segment an object from an image however, one needs closed region boundaries. The desired edges are the boundaries between such objects.

Segmentation methods can also be applied to edges obtained from edge detectors. Lindeberg and Li ${ }^{\left[{ }^{[8}\right.}$ developed an integrated method that segments edges into straight and curved edge segments for parts-based object recognition, based on a minimum description length (MDL) criterion that was optimized by a split-and-merge-like method with candidate breakpoints obtained from complementary junction cues to obtain more likely points at which to consider partitions into different segments.

\subsection{Method}

algorithms can be selected manually according to a priori knowledge or automatically by image information. These algorithms further divided to edge-based, region-based and hybrid. Edge-based algorithms are related with the edge information. The Structures of an object can be depicted by edge points. Common edge detection algorithms such as canny edge detector and Laplacian edge detector can be classified to this type of regions. These algorithms are used to find the edge pixels while eliminating the noise influence.

For example, canny edge detector used the threshold of gradient magnitude to find the potential edge pixels and suppressed them through the procedures of the non-maximal suppression and hysterics Thresholding. As the operations used in these algorithms are based on pixels, the detected edges are consisted of discrete pixels and hence may be incomplete or discontinuous. Hence, it must be apply the post processing like morphological operation to connect the breaks or eliminate the holes. This method has the ability that can be used to segment 3D image with good accuracy, but the disadvantage of this method is the difficulty to process the images of textured blob objects.

Image segmentation by is a simple but powerful approach for segmenting images having light objects on dark background [6]. technique is based on image space regions i.e. on characteristics of image [7]. operation convert a multilevel image into a binary image i.e., it choose a proper threshold $\mathrm{T}$, to divide image pixels into several regions and separate objects from background. Any pixel $(x, y)$ is considered as a part of object if its intensity is greater than or equal to threshold value i.e., $f(x, y) \geq T$, else pixel belong to background [7]. As per the selection of value, two types of methods are in existence [8], global and local Thresholding. When T is constant, the approach is called global otherwise it is called local Thresholding. Global methods can fail when the background illumination is uneven. In local Thresholding, multiple thresholds are used to compensate for uneven illumination [8]. Threshold selection is typically done interactively however; it is possible to derive automatic threshold selection algorithms.

Limitation of method is that, only two classes are generated, and it cannot be applied to multichannel images. In addition, does not take into account the spatial characteristics of an image due to this it is sensitive to noise [4], as both of these artifacts corrupt the histogram of the image, making separation more difficult.

\subsection{Region Based Segmentation Methods}

Compared to edge detection method, segmentation algorithms based on region are relatively simple and more immune to noise [7]. Edge based methods partition an image based on rapid changes in intensity near edges whereas region based methods, partition an image into regions that are similar according to a set of predefined criteria [9]. Segmentation algorithms based on region mainly include following methods:

\subsection{Segmentation Based On Clustering}

Clustering is an unsupervised learning task, where one needs to identify a finite set of categories known as clusters to classify pixels. Clustering use no training stages rather train themselves using available data. Clustering is mainly used when classes are known in advance. A similarity criteria is defined between pixels [1], and then similar pixels are grouped together to form clusters. The grouping of pixels into clusters is based on the principle of maximizing the intra class similarity and maximizing the inter class similarity. The quality of a clustering result depends on both the similarity measure used by the method and its implementation. Clustering algorithms are classified as hard clustering, k- means clustering, fuzzy clustering, etc.

\subsection{Region growing}

Region growing is a technique for extracting a region of the image that is connected based on some predefined criteria. This criteria can be based on intensity information and/or edges in the image. In its simplest form, region growing requires a seed point that is manually selected by an operator, and extracts all pixels connected to the initial seed with the same intensity value. 
Like thresholding, region growing is not often used alone but within a set of image processing operations, particularly for the delineation of small, simple structures such as tumors and lesions. Its primary disadvantage is that it requires manual in-traction to obtain the seed point. Thus, for each region that needs to be extracted, a seed must be planted. Split and merge algorithms are related to region growing but do not require a seed point . Region growing can also be sensitive to noise, causing extracted regions to have holes or even become disconnected. Conversely, partial volume effects can cause separate regions to become connected. To help alleviate these problems, a homotopic region growing algorithm has been proposed that preserves the topology between an initial region and an extracted region. Fuzzy analogies to region growing have also been developed.

\subsection{Classifiers}

Classifiers are known as supervised methods since they require training data that are manually segmented and then used as references for automatically segmenting new data. There are a number of ways in which training data can be applied in classifier methods. A simple classifier is the nearest-neighbor classifier, where each pixel or voxel is classified in the same class as the training datum with the closest intensity. The k-nearest-neighbor (kNN) classifier is a generalization of this approach, where the pixel is classified accord-ing to the majority vote of the $\mathrm{k}$ closest training data. The kNN classifier is considered a nonparametric classifier since it makes no underlying assumption about the statistical structure of the data. Another nonparametric classifier is the Parzen window, where the classification is made according to the majority vote within a predefined window of the feature space centered at the unlabeled pixel intensity.

A commonly-used parametric classifier is the maximum likelihood (ML) or Bayes classifier. It assumes that the pixel intensities are independent samples from a mixture of probability distributions, usually Gaussian. This mixture, called a finite mixture model, is given by the probability density function

$$
f\left(y_{j} ; \ldots ;\right)=X_{k=1}{ }_{k} f_{k}\left(y_{j} ; \_k\right)
$$

whereyj is the intensity of pixel $\mathrm{j}$, fk is a component probability density function parameterized by $\_\mathrm{k}$, and $=\left[\mathrm{L}_{1} ;::: ; \mathrm{K}\right]$. The variables_ $\mathrm{k}$ are mixing coefficients that weight the contribution of each density function and $\_=\left[1 ;::: ; \mathrm{K}_{-}\right]$. Training data is collected by obtaining representative samples from each component of the mixture model and then estimating each _k accordingly. For Gaussian mixtures, this means estimating $\mathrm{K}$ means, covariance's, and mixing coefficients. Classification of new data is obtained by assigning each pixel to the class with the highest posterior probability. When the data truly follows a finite Gaussian mixture distribution, the ML classifier can perform well and is capable of providing a soft segmentation composed of the posterior probabilities. Additional parametric and nonparametric classifiers are described in [14].

Standard classifiers require that the structures to be segmented possess distinct quantifiable features. Because training data can be labeled, classifiers can transfer these labels to new data as long as the feature space sufficiently distinguishes each label as well. Being non-iterative, they are relatively computationally efficient and unlike methods, they can be applied to multi-channel images. A disadvantage of classifiers is that they generally do not perform any spatial modeling. This weakness has been addressed in recent work extending classifier methods to segmenting images that are corrupted by intensity in homogeneities [10]. Neighborhood and geometric information were also incorporated into a classifier approach in [11]. Another disadvantage is the requirement of manual interaction for obtaining training data.

\subsection{Markov Random Field Models}

Markov random field (MRF) modeling itself is not a segmentation method but a statistical model which can be used within segmentation methods. MRFs model spatial interactions between neighboring or nearby pixels. These local correlations provide a mechanism for modeling a variety of image properties [12]. In medical imaging, they are typically used to take into account the fact that most pixels belong to the same class as their neighboring pixels. In physical terms, this implies that any anatomical structure that consists of only one pixel has a very low probability of occurring under a MRF assumption.

MRFs are often incorporated into clustering segmentation algorithms such as the K-means algorithm under a Bayesian prior model. The segmentation is then obtained by maximizing the a posteriori probability of the segmentation given the image data using iterative methods such as iterated conditional modes or simulated annealing.

\subsection{Artificial neural networks}

Artificial neural networks (ANNs) are massively parallel networks of processing elements or nodes that simulate biological learning. Each node in an ANN is capable of performing elementary computations. Learning is achieved through the adaptation of weights assigned to the connections between nodesANNs represent a paradigm for machine learning and can be used in a variety of ways for image segmentation. The most widely applied use in medical imaging is as a classifier, where the weights are determined using training data, and the ANN is then used to segment new data. ANNs can also be used in an unsupervised fashion as a clustering method as well as for deformable models [13].Because of the many interconnections used in a neural network, spatial information can easily be incorporated into its classification procedures. Although ANNs are inherently parallel, their processing is usually simulated on a standard serial computer, thus reducing this potential computational advantage. 


\begin{tabular}{|c|c|c|}
\hline $\begin{array}{l}\text { Segmentation } \\
\text { technique }\end{array}$ & Method description & Limitations \\
\hline $\begin{array}{l}\text { Region } \\
\text { growing }\end{array}$ & $\begin{array}{l}\text { Region growing is a technique for extracting a } \\
\text { region of the image that is connected based on } \\
\text { some predefined criteria. This criteria can be } \\
\text { based on intensity information and/or edges in } \\
\text { the image... }\end{array}$ & $\begin{array}{l}\text { Its primary disadvantage is that it } \\
\text { requires manual in-traction to obtain the } \\
\text { seed point. Thus, for each region that } \\
\text { needs to be extracted, a seed must be } \\
\text { planted. }\end{array}$ \\
\hline Classifiers & $\begin{array}{l}\text { Classifiers are known as supervised methods } \\
\text { since they require training data that are } \\
\text { manually segmented and then used as } \\
\text { references for automatically segmenting new } \\
\text { data. }\end{array}$ & $\begin{array}{l}\text { A disadvantage of classifiers is that } \\
\text { they generally do not perform any } \\
\text { spatial modeling. This weakness has } \\
\text { been addressed in recent work } \\
\text { extending classifier methods to } \\
\text { segmenting images that are corrupted } \\
\text { by intensity in homogeneities }\end{array}$ \\
\hline $\begin{array}{l}\text { Clustering } \\
\text { Approach }\end{array}$ & $\begin{array}{l}\text { Assumes that each region in the image forms a } \\
\text { separate cluster in the feature space. Can be } \\
\text { generally broken into two steps(1) categorize } \\
\text { the points in the feature space into } \\
\text { clusters;(2)map the clusters back to the spatial } \\
\text { domain to form separate regions }\end{array}$ & $\begin{array}{l}\text { (1)How to determine the number of } \\
\text { clusters (known as cluster validity) } \\
\text { (2) Features are often image dependent } \\
\text { and how to select features so as to } \\
\text { obtain } \\
\text { satisfactory segmentation results } \\
\text { remains unclear } \\
\text { (3) Does not utilize spatial Information }\end{array}$ \\
\hline Method & $\begin{array}{l}\text { Requires that the histogram of an image } \\
\text { has a number of peaks, each corresponds } \\
\text { to a region }\end{array}$ & $\begin{array}{l}\text { (1) Does not work well for an image } \\
\text { without any obvious peaks or with } \\
\text { broad and flat } \\
\text { valleys. } \\
\text { (2) Does not consider the spatial } \\
\text { details, so cannot guarantee that the } \\
\text { segmented regions are contiguous }\end{array}$ \\
\hline $\begin{array}{l}\text { Region-based } \\
\text { Approaches }\end{array}$ & $\begin{array}{l}\text { Group pixels into homogeneous regions. } \\
\text { Including region growing, region } \\
\text { splitting, region merging or their } \\
\text { combination }\end{array}$ & $\begin{array}{l}\text { (1) Are by nature sequential and quite } \\
\text { expensive both in computational time } \\
\text { and memory (2) Region growing has } \\
\text { inherent } \\
\text { dependence on the selection of seed } \\
\text { region and the order in which pixels and } \\
\text { regions are }\end{array}$ \\
\hline $\begin{array}{l}\text { Edge } \\
\text { detection } \\
\text { approaches }\end{array}$ & $\begin{array}{l}\text { Based on the detection of discontinuity, } \\
\text { normally tries to locate points with more } \\
\text { or less abrupt changes in gray level. } \\
\text { Usually classified into two categories: } \\
\text { sequential and parallel }\end{array}$ & $\begin{array}{l}\text { (1) Does not work well with images in } \\
\text { which the edges are ill-defined or there } \\
\text { are too many edges } \\
\text { (2) It is not a trivial job to produce a } \\
\text { closed curve or boundary } \\
\text { (3) Less immune to noise than other } \\
\text { techniques, e.g., and clustering }\end{array}$ \\
\hline
\end{tabular}

TABLE 1: COMPARISON OF IMAGE SEGMENTATION TECHNIQUES

\section{CONCLUSION}

In this paper various image segmentations mythologies and technique applied for medical image processing are briefly explained and overviewed. The study also focuses on different research issues In this field and methodologies applied for medical image segmentation. Thestudy focuses to guide that researcher who carried out their research in medical image segmentation. Inspired of several decades of research in this field, Medical segmentation has a promising future as its focus on contemporary research. Thisbecausethere is no universally accepted 
methodologies for image segmentation as their results and various conclusion a fact factor likes spatial characteristic of image continuously ,contain ,texture, edges and homogeneity of images. Due to all above factors there is no singlemethod which can be considered as a universal methodology for all time of images and hence image segmentation is still a challenging problem in the field of image processing.

\section{REFERENCES}

1.Zhang, Y. J, An Overview of Image and Video Segmentation in the last 40 years, Proceedings of the $6^{\text {th }}$ International Symposium on Signal Processing and Its Applications, pp. 144-151, 2001.

2.Wahba Marian, An Automated Modified Region Growing Technique for Prostate Segmentation in Trans-Rectal Ultrasound Images, Master's Thesis, Department of Electrical and Computer Engineering, University of Waterloo, Waterloo, Ontario, Canada, 2008.

3. P.K. Sahoo, S. Soltani, and A.K.C. Wong.A survey of techniques. Comput. Vis. Graph.Im. Proc., 41:233 $260,1988$.

4.N. R. Pal, S. K. Pal,“A Review on Image Segmentation n Techniques”, Pattern Recognition, Vol. 26, No. 9, pp. 1277- 1294, 1993.

5.W. X. Kang, Q. Q. Yang, R. R. Liang,“TheComparativee Research on Image Segmentation Algorithms”, IEEE Conference on ETCS, pp. 703-707, 2009.

6.Rastgarpour M., and Shanbehzadeh J., Application of Al Techniques in Medical Image Segmentation and Novel Categorization of Available Methods and Tools, Proceedings of the International MultiConference of Engineers and Computer Scientists 2011 Vol I, IMECS 2011, March 16-18, 2011, Hong Kong.

7. L.Aurdal,"Image Segmentation beyond thresholding”, NorskRegnescentral, 2006.

8. Y. Zhang, H. Qu, Y. Wang,"Adaptive Image Segmentati on Based on Fast and Image Merging”, Artificial reality and Telexistence-Works hops, pp. 308-311, 1994.

9.H. G. Kaganami, Z. Beij,"Region Based Detection versus Edge Detection”, IEEE Transactions on Intelligent information hiding and multimedia signal processing, pp. 1217-1221, 2009.

10.W.M. Wells, W.E.L. Grimson, R. Kikins, and F.A. Jolesz. Adaptive segmentation of MRI data. IEEE

T. Med. Imag., 15:429-442, 1996.

11. T. Kapur, W.E.L. Grimson, R. Kikinis, and W.M. Wells.Enhanced spatial priors for segmentation of magnetic resonance imagery.In Proc. 1st Int. Conf. Med. Im.Comput. Comp. Assist. Interven.

(MICCA198), pages 457-468, 1998.

12.S.Z. Li. Markov random field modeling in computer vision. Springer, 1995.

13. D.L. Vilarino, V.M. Brea, D. Cabello, and J.M. Pardo. Discrete-time CNN for image segmentation by active contours. Patt. Rec. Let., 19:721-734, 1998.

14. A.P. Zijdenbos and B.M. Dawant.Brain segmentation and white matter lesion detection in MR im-ages.Critical Reviews in Biomedical Engineering, 22:401-465, 1994. 